\title{
Poisson's Ratio Evaluation of LPCVD Silicon Nitride Film
}

\author{
Member Osamu Tabata (Toyota Central R \& D Labs., Inc.) \\ Toshiyuki Tsuchiya (Toyota Central R \& D Labs., Inc.)
}

Key words : Poisson's ratio, Thin film, Silicon nitride, Ion implantation

\section{Introduction}

Micro ElectroMechanical Systems (MEMS) take advantage of thin films as structural materials. With increasing interest in MEMS, the importance of mechanical properties of thin films came to be recognized. Evaluation and understanding of mechanical properties of thin films are especially necessary for designing of MEMS. Since thin films are difficult to handle, special techniques are required to evaluate theirmechanical properties. Several techniques using siliconmicromachined structure have been proposed, and they make it possible to evaluate internal stress, Young's modulus, friction coefficient and fracture strength. However, there are few reports on the evaluation of Poisson's ratios of thin films. Evaluation of thin film Poisson's ratio and Young's modulus has a great meaning from the viewpoint of material characterization. This is because the elastic behavior of an isotropic material can be completely defined by these two constants.

This paper reports a new technique for evaluating Poisson's ratio $v$ of a thin film. The evaluation technique utilizes the pressure-deflection technique of rectangular membrane structure ${ }^{(1)}$ that we previously proposed as a Young's modulus measurement technique. The principle and the results of the measured $v$ for LPCVD silicon nitride are described.

\section{Procedure of Poisson's Ratio Evaluation}

The cross sectional view of the sample subjected to the measurement is shown in Fig. 1. The sample is prepared by depositing the object thin film on a silicon substrate and subsequent anisotropic etching of a part of the silicon substrate. The fabricated membranes are rectangular in profile having different ratios $n$ of the shorter to the larger sides. The membranes are a few $\mathrm{mm}$ in size and 1 Hm or less in thickness.

The deflection of the membrane can be related to the pressure as expressed by Eq. (1);

$$
p=\sigma C_{1}(n) \frac{t}{a^{2}} h+E C_{2}(n, v) \frac{t}{a^{4}}
$$

where $p$ and $h$ are the pressure applied to the membrane and the deflection at the central point of the membrane, respectively, $E$ is Young's modulus, $\sigma$ is the internal stress and $t$ is the thickness of the film. $C_{,}(n)$ is a constant defined by $n$ and $C_{2}(n, v)$ is a constant defined by $n$ and $v$ of the film.

The constants $C_{1}(n)$ and $C_{2}(n, v)$ which appeared in Eq. (1) can be analytically derived using energy minimization method by assuming the deflected membrane shape. ${ }^{17}$ However, the derived constants are in disagreement with experimental values for a square membrane as pointed out by Pan. ${ }^{(2)}$ Therefore, we made FEM analysis of the pressuredeflection relationship of a rectangularmembrane to obtain an accurate value of $C_{2}(n, v)$. Finite element modeling of the rectangular membrane was carried out using NASTRAN. A quarter of the membrane was modeled by using 4 -node shell elements. First, the deflection was calculated for assumed values; namely membrane length of shorterside of $2 \mathrm{~mm}$, a membrane thickness of $0.2 \mu \mathrm{m}$, internal stress of 0 , Young's modulus of $300 \mathrm{GPa}$ and an applied pressure of $3 \mathrm{~Pa}$ for different values of membrane length of longer sides from $2 \mathrm{~mm}$ to infinite, and $v$ from 0.05 to 0.45 . From the calculated deflection value, we determined the value $C_{2}(n, v)$ for various $n$ 's and $v$ 's. Dependencies of $C_{2}(n, v)$ on $v$ for various $n$ 's of 1, 1.5, 2, 4 and infinite are shown in Fig. 2.

Using a dependence of $C_{2}(n, v)$ on $v, v$ can be defined as follows:

(1) Square and rectangular membranes are fabricated.

(2) Pressure-deflection relationships of the two membranes are measured.

(3) $E C_{2}(l, v)$ for the square membrane and $E C_{2}(n, v)$ for the rectangular membrane are calculated by fitting Eq. (1) to the measured pressure-deflection relationships. Although the values $E$ and $C_{2}(n, v)$ are unknown at this time, the multiplied values $E C_{2}(l, v)$ and $E C_{2}(n, v)$ can be obtained from the measured

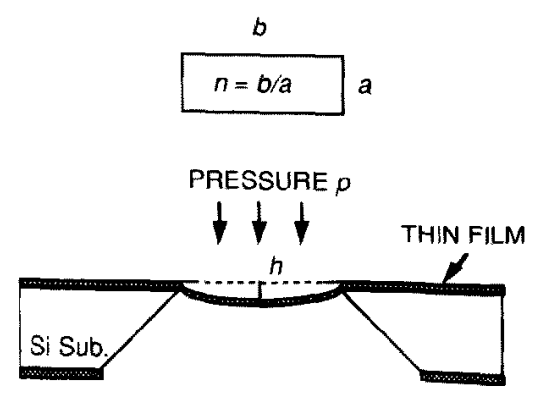

Fig.1 Cross-section of a sample. 


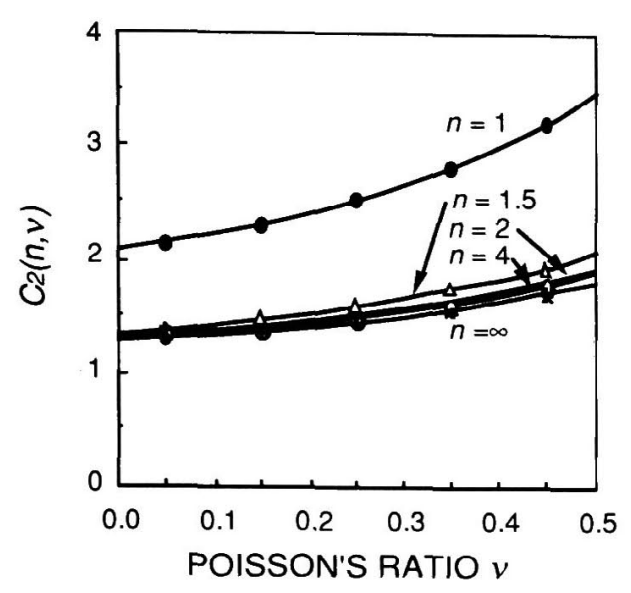

Fig.2 Dependency of $C_{2}(n, v)$ on Poisson's ratio $v$ for various values of $n$.

data.

(4) The ratio $C_{2}(1, v) / C_{2}(n, v)$ is calculated by dividing $E C_{2}(1, v)$ by $E C_{2}(n, v)$.

(5) The dependency of $C_{2}(1, v) / C_{2}(n, v)$ on $v$ can be calculated from the FEM results shown in Fig. 2 in advance. Figure 3 shows the calculated dependencies of $C_{2}(l, v) / C_{2}(n, v)$ on $v$ for $n=1.5$, 2,4 and infinite. Therefore, $v$ can be defined from the measured $C_{2}(I, v) / C_{2}(n, v)$.

\section{Experimental Results}

The proposed technique was applied to the $v$ evaluation of a LPCVD silicon nitride thin films without and with boron ion implantation of $4 \times 10^{15} / \mathrm{cm}^{2}, 30 \mathrm{keV}$ to control internal stress of the film. ${ }^{(1)}$ The two membranes, a $2 \mathrm{~mm} \times 2 \mathrm{~mm}$ square one and a $2 \mathrm{~mm} \times$ $8 \mathrm{~mm}$ rectangular one with a thickness of $0.2 \mu \mathrm{m}$, were used. More than ten samples were used for the measurements of pressuredeflection relationships of the membranes without and with ion implantation. By fitting Eq. (1) to the measured pressure-deflection relationships, $E C_{2}(1, v)$ and $E C_{2}(4, v)$ were calculated. Each of these values is averaged for the membranes without and with ion implantation, and $C_{2}(1, v) / C_{2}(4, v)$ was calculated by using the averaged value for each of the films without and with ion implantation. The $v$ 's for the films without and with ion implantation were obtained successfully to be 0.35 and 0.33 by using the calculated $C_{2}(1, v) / C_{2}(4, v)$ values of 1.824 and 1.809 , respectively. The obtained $v$ values of 0.35 and 0.33 are larger than those of sintered silicon nitride ceramics. This difference may be caused by the structure difference between amorphous LPCVD silicon nitride and polycrystalline sintered silicon nitride. Further investigation should be carried out to clarify the evaluation accuracy.

\section{Conclusion}

In summary, a technique for evaluating Poisson's ratio $v$ of a thin film has been proposed. This technique utilizes the pressure-deflection

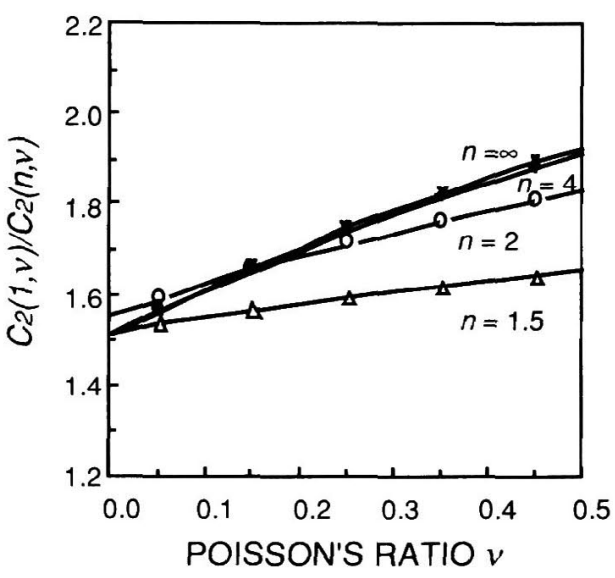

Fig.3 Dependency of $C_{2}(1, v) / C_{2}(n, v)$ on Poisson's ratio $v$. relationships of square and rectangular membranes. The technique has been applied to the $v$ evaluation for LPCVD silicon nitride thin film without and with boron ion implantation of $4 \times 10^{15} / \mathrm{cm}^{2}, 30 \mathrm{keV}$, and $v$ values of 0.35 and 0.33 were determined, respectively.

(Received 22 Aug 1995)

\section{REFERENCES}

(1) O. Tabata, K. Kawahata, S. Sugiyama and I. Igarashi: "Mecahnical Property Measurements of Thin Films Using Load-Deflection of Composite Rectangular Membranes", Sensors and Actuators, 20, 135 141 (1989)

(2) J. Y. Pan, P. Lin, F. Maseeh and S. D. Senturia: "Verification of FEM Analysis of Load-Deflection Methods for Measuring Mechanical Properties of Thin Films", Tech. Digest, IEEE SolidState Sensors and Actuators Workshop, 70 73 (1990)

Osamu Tabata (Member) Osamu Tabata received the M.S.

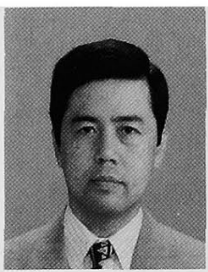
degree and the Ph.D. degree from Nagoya Institute of Technology in 1981 and in 1993, respectively. Since 1981 , he has been working for Toyota Central R \& D Labs., Inc. and is currently engaged in the research and development of silicon sensors, MEMS, silicon micromachining and thin film mechanical property.

He was honored with the Science News Award in 1987, Presentation Paper Award in 1992 and R\&D 100 Award in 1993.

Toshiyuki Tsuchiya Toshiyuki Tsuchiya received the M.S.

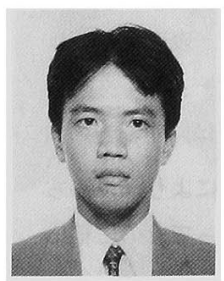
degree from University of Tokyo in 1993. In 1993, he joined Toyota Central R \& D Labs., Inc., where he is currently engaged in the research of silicon micromachining and thin film mechanical property. 